\title{
Precise half-life measurement of the ${ }^{26} \mathrm{Si}$ ground state
}

I. Matea ${ }^{1, a}$, J. Souin ${ }^{1,2}$, J. Äystö ${ }^{3}$, B. Blank ${ }^{1}$, P. Delahaye ${ }^{4}$, V.-V. Elomaa ${ }^{3}$, T. Eronen ${ }^{3}$, J. Giovinazzo $^{1}$, U. Hager $^{3, b}$, J. Hakala ${ }^{3}$, J. Huikari ${ }^{1}$, A. Jokinen ${ }^{3}$, A. Kankainen ${ }^{3}$, I.D. Moore ${ }^{3}$, J.-L. Pedroza ${ }^{1}$, S. Rahaman ${ }^{3}$, J. Rissanen ${ }^{3}$, J. Ronkainen ${ }^{3}$, A. Saastamoinen ${ }^{3}$, T. Sonoda ${ }^{3, c}$, and C. Weber ${ }^{3}$

1 Centre d'Études Nucléaires de Bordeaux Gradignan - Université Bordeaux 1 - UMR 5797 CNRS/IN2P3, Chemin du Solarium, BP 120, F-33175 Gradignan Cedex, France

2 Instituto Estructura de la Materia, CSIC, Serrano 113bis, E-28006 Madrid, Spain

3 Department of Physics, University of Jyväskylä, P.O. Box 35, FI-40014, Jyväskylä, Finland

4 CERN, CH-1211 Geneva 23, Switzerland

Original article: Eur. Phys. J. A 37, 151 (2008) DOI: 10.1140/epja/i2008-10623-5

Received: 29 October 2008

Published online: 12 November 2008 - (c) Società Italiana di Fisica / Springer-Verlag 2008

In [1] we have reported the measurement of the $\beta$-decay half-life of ${ }^{26} \mathrm{Si}$ with a relative precision better than $2 \cdot 10^{-3}$. In table 3 and in the text on p. 157, the error of the branching ratio for the analogue and the non-analogue transitions of ${ }^{26} \mathrm{Si}$ should be $0.58 \%$ and not $2.32 \%$. As a consequence, the average value of all published data for the super-allowed branching ratio is $75.48(58) \%$. With this value we obtain $f t=3024(26) \mathrm{s}$ and $\mathcal{F} t=3047(26)$ s. The new table is given below, slightly modified.

Table 3. The absolute $\beta^{+}$BR for the most intense $\gamma$-line, $829 \mathrm{keV}(\mathrm{BR}(1058 \mathrm{keV}))$, and the relative intensity of the $1622 \mathrm{keV}$ transition with respect to the $829 \mathrm{keV}$ line $\left(\gamma_{1622} / \gamma_{829}\right)$ are reported for each germanium detector and compared with the adopted values in [2]. Using the average values from the present table for the $829 \mathrm{keV}$ and the $1622 \mathrm{keV}$ lines and the intensities for the non-observed lines from [2], we find a total non-analogue branching ratio of $24.52(58) \%$ and thus an analogue branching ratio of $75.48(58) \%$.

\begin{tabular}{lrrrrr}
\hline & Ge1 & Ge2 & Ge3 & Mean values & {$[2]$} \\
\hline $\mathrm{BR}(1058 \mathrm{keV})(\%)$ & $21.03(94)$ & $20.15(73)$ & $22.19(67)$ & $21.21(64)$ & $21.8(8)$ \\
$\gamma_{1622} / \gamma_{829}$ & & & & $0.1301(62)$ & $0.1265(36)$ \\
\hline
\end{tabular}

\section{References}

1. I. Matea et al., Eur. Phys. J. A 37, 151 (2008).

2. J.C. Hardy, I.S. Towner, Phys. Rev. C 71, 055501 (2005).

\footnotetext{
a e-mail: matea@cenbg.in2p3.fr

b Present address: TRIUMF, 4004 Wesbrook Mall, Vancouver, British Columbia, V6T 2A3, Canada.

c Present address: Instituut voor Kern- en Stralingsfysica, Celestijnenlaan 200D, B-3001 Leuven, Belgium.
} 